\title{
UNSTEADY DIFFUSION FROM A SOURCE IN UNIFORM STREAM
}

\author{
BY \\ C. Y. WANG \\ Michigan State University, East Lansing, Michigan
}

\begin{abstract}
Basic exact solutions for an unsteady point heat source in a uniform stream are presented. The governing equation is solved using changes of variables and Laplace transform. Both diffusion and convection are important. The periodic source sheds a trail of thermal energy packets, alternating and rapidly decreasing in strength. The suddenly started source reaches steady state in a time of 4 (diffusivity) $/(\text { velocity })^{2}$.
\end{abstract}

Introduction and formulation. Convection and diffusion are the basic mechanisms underlying heat, mass, and momentum transfer. It seems, however, there are very few exact solutions for unsteady convection-diffusion problems [1,2]. Literature in this area either approximated the problem by assumptions such as boundary layer theory or directly integrated the governing partial differential equations numerically. In this note we shall introduce some simple exact solutions to the unsteady diffusionconvection equation.

Consider a point source in a uniform stream. The governing equation is

$$
T_{t^{\prime}}^{\prime}+U T_{x^{\prime}}^{\prime}=a\left(T_{x^{\prime} x^{\prime}}^{\prime}+T_{y^{\prime} y^{\prime}}^{\prime}+T_{z^{\prime} z^{\prime}}^{\prime}\right) .
$$

Here, $T^{\prime}$ is the temperature (concentration) difference, $U$ is the constant free stream velocity, and $a$ is diffusivity. We normalize all lengths by $2 a / U$, the time by $1 / \omega$, where $\omega$ is some frequency scale, and drop primes. Eq. (1) becomes

$$
\left(\frac{2 \omega a}{U^{2}}\right) T_{t}^{\prime}+T_{x}^{\prime}=\frac{1}{2}\left(T_{x x}^{\prime}+T_{y y}^{\prime}+T_{z z}^{\prime}\right) .
$$

Set

$$
T^{\prime}=c e^{x} \phi(x, y, z, t),
$$

where $c$ is a constant to be determined. Eq. (2) further reduces to

$$
\left(\frac{4 \omega a}{U^{2}}\right) \phi_{t}+\phi=\nabla^{2} \phi .
$$

Received August 7, 1990. 
Let the heat (mass) flux from the point source at the origin be a given function of time $q(t)$; thus

$$
q(t)=\lim _{r^{\prime} \rightarrow 0}\left(-4 \pi r^{\prime 2} k \frac{\partial T^{\prime}}{\partial r^{\prime}}\right) .
$$

Here, $k$ is the conductivity and $\left(r^{\prime}, \theta\right)$ are spherical coordinates:

$$
r^{\prime}=\frac{2 a}{U} r=\sqrt{\left(x^{\prime}\right)^{2}+\left(y^{\prime}\right)^{2}+\left(z^{\prime}\right)^{2}}, \quad x^{\prime}=r^{\prime} \cos \theta .
$$

Eq. (5) yields

$$
\lim _{r \rightarrow 0} \phi_{r}(r, \theta, t)=\frac{-U}{8 \pi a k c} \frac{q(t)}{r^{2}} .
$$

Another boundary condition is the decay of temperature difference at infinity,

$$
\lim _{r \rightarrow \infty} e^{r \cos \theta} \phi(r, \theta, t)=0 .
$$

From the boundary conditions and Eq. (4), we conclude $\phi$ must be a function of $(r, t)$ only. Thus,

$$
\phi(r, t)+\lambda \phi_{t}=\frac{1}{r^{2}}\left(r^{2} \phi_{r}\right)_{r},
$$

where $\lambda \equiv 4 \omega a / U^{2}$. Eqs. (7)-(9) are to be solved with given $q(t)$.

Periodic source. In this case

$$
q(t)=\mathscr{R} e q_{0} e^{i \omega t^{\prime}},
$$

where $q_{0}$ is the strength amplitude. We set

$$
c=\frac{q_{0} U}{8 \pi a k}
$$

and Eq. (7) becomes

$$
\lim _{r \rightarrow 0} \phi_{r}=-\frac{e^{i t}}{r^{2}}
$$

Let

$$
\phi(r, t)=\frac{g(r)}{r} e^{i t}
$$

Eq. (9) simplifies to

$$
(1+i \lambda) g=g_{r r} .
$$

The solution, satisfying $g(0)=1$ and $\lim _{r \rightarrow \infty} e^{r} g / r=0$, is

$$
g=\exp \left[-\left(1+\lambda^{2}\right)^{1 / 4} \exp \left(\frac{i}{2} \tan ^{-1} \lambda\right) r\right] \text {. }
$$

The normalized temperature distribution $T$ is

$$
\begin{aligned}
T=\frac{T^{\prime}}{c}=\mathscr{R} e e^{x} \phi(r, t)= & \frac{1}{r} \exp \left[r \cos \theta-r\left(1+\lambda^{2}\right)^{1 / 4} \cos \left(\frac{1}{2} \tan ^{-1} \lambda\right)\right] \\
& \cdot \cos \left[t-r\left(1+\lambda^{2}\right)^{1 / 4} \sin \left(\frac{1}{2} \tan ^{-1} \lambda\right)\right] .
\end{aligned}
$$


When the frequency $\omega$ (and thus $\lambda$ ) becomes zero, the steady state temperature distribution $[3,4]$ is recovered,

$$
T=\frac{1}{r} e^{x-r}
$$

As $\lambda$ increases, the phase shift increases and the amplitude rapidly decreases. Also convection effects, represented by $\exp (r \cos \theta)$, become less dominant. Fig. 1 (see p. 52) shows the constant temperature lines at various times. A series of thermal concentrations, altering in sign, are convected downstream. Due to diffusion, their strength decreases with distance.

Sudden constant course. In this case

$$
q(t)= \begin{cases}q_{0}, & t>0 \\ 0, & t<0\end{cases}
$$

Since there is no natural time scale we set $\omega=U^{2} /(4 a)$ and define the Laplace transform

$$
f(r, s)=\mathscr{L} \phi=\int_{0}^{\infty} e^{-s t} \phi(r, t) d t, \quad s>0 .
$$

The governing equations become

$$
\begin{gathered}
(1+s) f=\frac{1}{r^{2}}\left(r^{2} f_{r}\right)_{r}, \\
\lim _{r \rightarrow 0} f_{r}=\frac{-1}{s r^{2}}, \\
\lim _{r \rightarrow \infty} f<O\left(e^{-r}\right) .
\end{gathered}
$$

Here the constant $c$ is defined as in Eq. (11). The solution is

$$
f=\frac{1}{s r} e^{-\sqrt{1+s} r} \text {. }
$$

The Laplace inversion is

$$
\begin{aligned}
\phi(r, t) & =\mathscr{L}^{-1} f(r, s)=\frac{1}{r} \mathscr{L}^{-1}\left(\frac{1}{s} e^{-\sqrt{1+s} r}\right) \\
& =\frac{1}{2 \sqrt{\pi}} \int_{0}^{t} t^{-3 / 2} \exp \left(-t-\frac{r^{2}}{4 t}\right) d t .
\end{aligned}
$$

The integral in Eq. (24) unfortunately cannot be evaluated in closed form. One can show, using the final value theorem, that

$$
\phi(r, \infty)=\frac{1}{r} e^{-r} .
$$

This agrees with the steady state solution Eq. (17).

For small times and $r=O(1)$ one can approximate the integral as follows. Define

$$
s=t+\frac{r^{2}}{4 t} \gg 1, \quad t=\frac{1}{2}\left(s-\sqrt{s^{2}-r^{2}}\right) \ll 1 .
$$



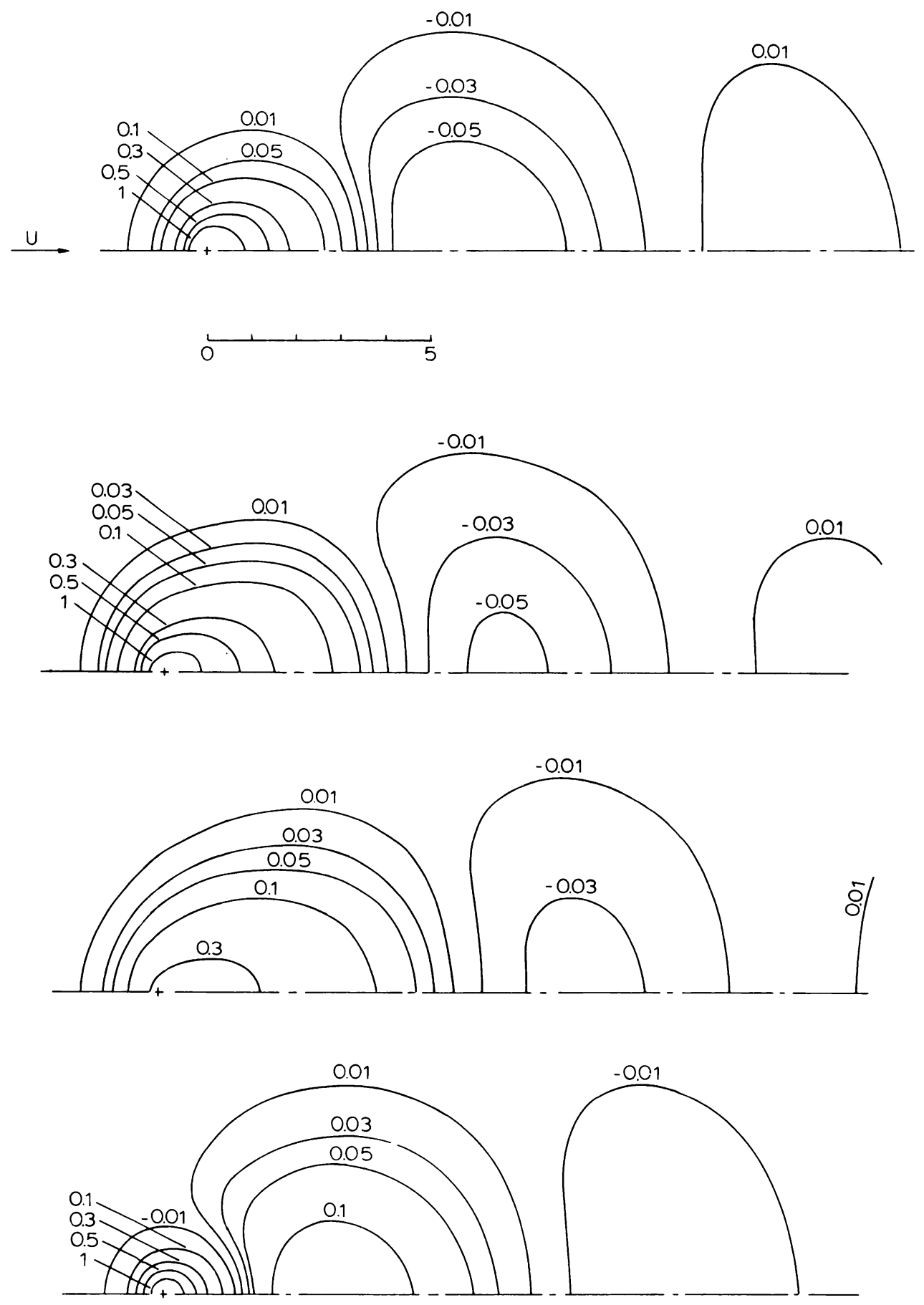

FIG. 1. Periodic source in uniform stream, $\lambda=1$. Lines shown are for constant normalized temperature $T$ (upper half only). (a) $t=0$, (b) $t=\pi / 4$, (c) $t=\pi / 2$, (d) $t=3 \pi / 4$. 
In terms of $s$, Eq. (24) becomes

$$
\phi(r, t)=\frac{1}{2 \sqrt{\pi}} \int_{\infty}^{s(t)}\left(\frac{s-\sqrt{s^{2}-r^{2}}}{2}\right)^{-3 / 2} e^{-s}\left(1-\frac{s}{\sqrt{s^{2}-r^{2}}}\right) d s
$$

Expanding in a Taylor series for large $s$ and integrating by parts give

$$
\begin{aligned}
2 \sqrt{\pi} \phi(r, t) & \sim \frac{2}{r} \int_{s}^{\infty} \frac{e^{-s}}{\sqrt{s}}\left[1+\frac{3}{8} \frac{r^{2}}{s^{2}}+O\left(s^{-4}\right)\right] d s \\
& =\frac{2 e^{-s}}{r \sqrt{s}}\left[1-\frac{1}{2} s^{-1}+\left(\frac{3}{8} r^{2}+\frac{3}{4}\right) s^{-2}-\left(\frac{15}{16} r^{2}+\frac{15}{8}\right) s^{-3}+O\left(s^{-4}\right)\right] .
\end{aligned}
$$

For general $r$ and $t$, the value of $\phi$ can be numerically integrated from Eq. (24). It is more advantageous to make the change of variable

$$
v=1-e^{-t}, \quad v \text { in }[0,1] \text {. }
$$

Then

$$
2 \sqrt{\pi} \phi=\int_{0}^{v(t)}[-\ln (1-v)]^{-3 / 2} \exp \left[\frac{r^{2}}{4 \ln (1-v)}\right] d v .
$$

The results through quadrature by Simpson's rule are shown in Fig. 2. We see that

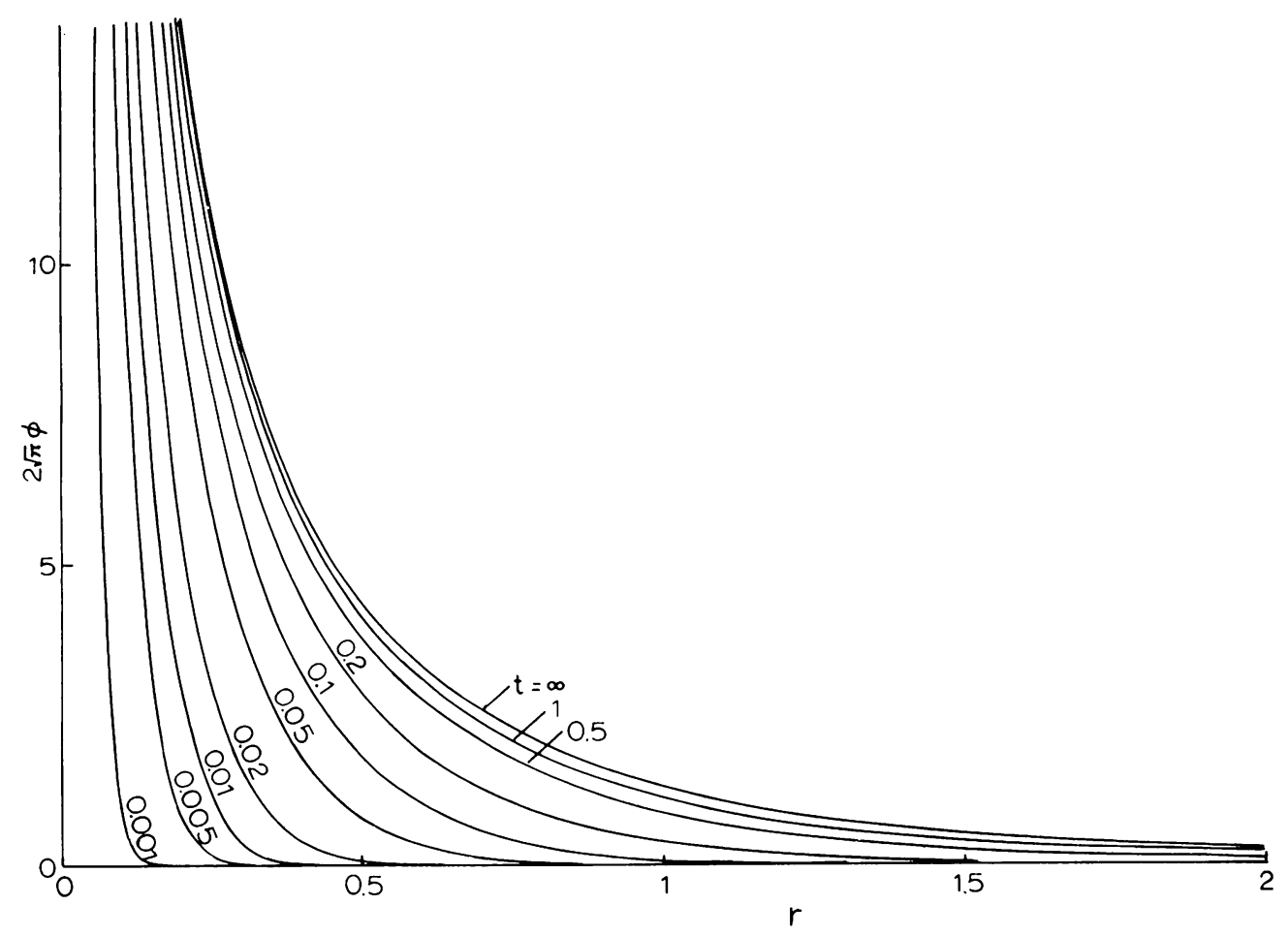

FIG. 2. The integral $2 \sqrt{\pi} \phi$ as a function of $r$ for various constant $t$. 

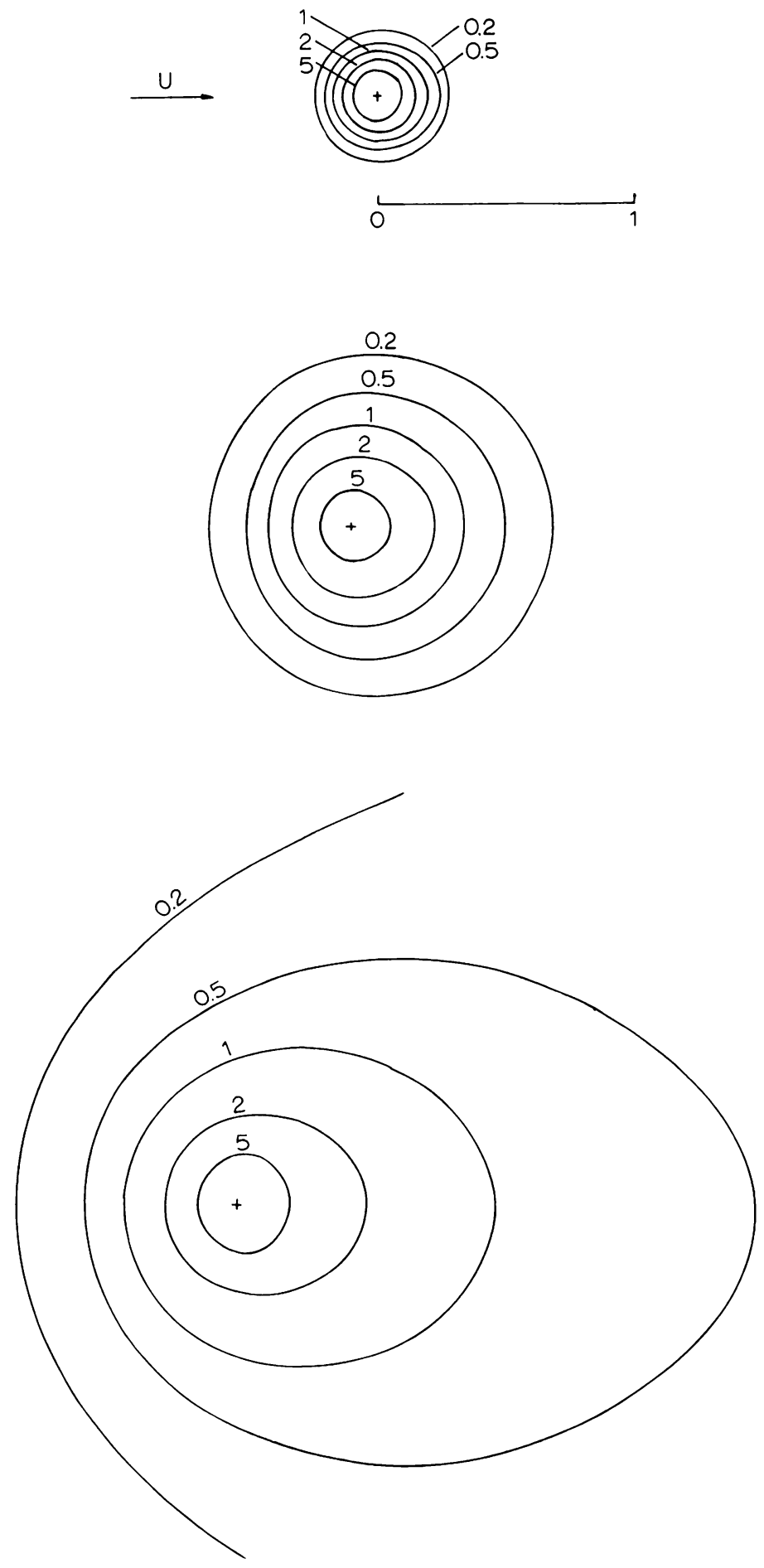

FIG. 3. Constant $T$ lines for a sudden constant source in uniform stream. (a) $t=0.01$, (b) $t=0.1$, (c) $t=\infty$ (Eq. 17). 
for $t>1$, steady state is effectively attained. Our approximate solution Eq. (28) is accurate for small $t$ and small $\phi$.

Using Fig. 2, the temperature distributions at various times after the introduction of the step source are shown in Fig. 3. In the beginning diffusion dominates. A balance between diffusion and convection is reached when $t>1$ or $t^{\prime}>4 a / U^{2}$.

Since the governing equation is linear, we may be able to superpose solutions. Thus, if the source strength is suddenly turned off at $t=t_{1}$, the solution is

$$
T= \begin{cases}0, & t<0, \\ \phi(r, t) \exp (r \cos \theta), & 0<t<t_{1}, \\ {\left[\phi(r, t)-\phi\left(r, t-t_{1}\right)\right] \exp (r \cos \theta),} & t_{1}<t .\end{cases}
$$

We expect a packet of thermal energy transported and diffused downstream.

Conclusions. This note presents, for the first time, two important exact solutions for an unsteady point source in a uniform stream. Our results show diffusion, including axial diffusion which is ignored in boundary layer theory, is quite dominant in unsteady problems. Also the quasi-steady assumption could not be valid if the time scale is less than $4 a / U^{2}$. The exact solutions presented here also serve as standards for testing numerical schemes involving diffusion and convection.

\section{REFERENCES}

[1] A. V. Liukov, Analytical Heat Diffusion Theory, Academic Press, New York, 1968

[2] J. Crank, The Mathematics of Diffusion, 2nd ed., Clarendon Press, Oxford, 1975

[3] D. Rosenthal, The theory of moving sources of heat and its application to metal treatments, Trans. ASME 68, 849-866 (1946)

[4] M. Jacob, Heat Transfer, Vol. I, Wiley, New York, 1949 